CROSSROADS

\title{
Exploring the Possibility for a Common System for Joint Aeromedical Standards
}

Justin Woodson*, Walter M Dalitsch III, James L Persson, James McGhee, Charles Ciccone, Brian Parsa

ABSTRACT: The Physical qualification standards for aviation service used by the United States Army, Navy/Marine Corps, Air Force, and Coast Guard developed in parallel, diverging in many instances due to differences ranging from terminology to mission. Presently, standards and requirements for waiver vary widely between the services, in spite of minimal differences in aeromedical concerns for any given medical condition. Standardization or increased concordance between the services would have several advantages leading to more efficient and effective delivery of aviation medical support to the operational forces. This is particularly true in an increasingly joint operational environment. The authors have identified four major hurdles that must be overcome before the concept of joint aviation physical standards can be explored. These include. a difference in terminology including aviator the prication, a diference in mission dentions and requirements, a diference in of thoce policies. Thy hurcles are axplord, and suggestiovs for their mitigation are presented with open discussion following.

Keywords: Aerospace Medicine, Aviation Medicine, Physical Standards, Military Medicine

INTRODUCTION

The clinical practice of Aviation Medicine in the U.S. Military revolves around an administrative landscape of aeromedical policy and physical standards which are specific to the four main branches of the U.S. Armed Forces including the U.S. Air the U. C. Amy, U.S. NavylMarine Corps, and ronment, military Flight Surgeons are increanily practicing in a joint miltary environment in which service members from different services must collaborate in order to accomplish the mission. In this joint military environment Flight Surgeons are expected to be equally well versed in the policies and procedures of their sister services, as they provide *To whom correspondence should be addressed
Dr. Justin Woodson LTC MC SFS US Ary Dr. Iustin Woodson LTC, MC, SFS, US Army
Uniformed Services University of Health Sciences Bethesda, MD

Email justin.woodson@us.armymil aeromedical care for aviators and aircrew members from all services. Despite commonalities in aeromedical concerns, each service's aeromedical policies have diverged over time, resulting in an ofen confusing and unnecessarily complicated join framework for aviation physical standards. There is increasing pressure from high levels of U.S. Depractices across services in many ares of support including but not limited to many areas of support paper explores the hurdles encountered in developing a set of joint Aeromedical physical standards and administrative procedures and proposes potential solutions to some of these problems. While these proposals are not intended to be comprehensive in nature, they are presented to raise awareness and initiate dialog between administrators throughout the aeromedical communities, with the goal of moving toward the authors' vision of a single common system of aeromedical administration for the U.S. Military. The scope of this article is limited to the U.S. Military for the sake of brevity, but parallel analysis with our international brethren is invited, as many of the same lessons clearly apply in the international military aviation medicine community.

BACKGROUND

The idea of a unified approach to medical service for all branches of the U.S. Armed Forces is not new. With four separate medical departments in the U.S. Navy/Marine Corps, U.S. Army, U.S. Air Force, and U.S. Coast Guard, the efficient delivery of health care to armed service members and their dependents has long been complicated by stovepiping of resources and programs. One of the most of Defen a medical services was pu Toth by Major General Norman Kirk in 1947. While Kirk did not originate this idea he materialized the concept in a detailed plan that he presented to the Senate Armed Forces Committee (13). Since that time several more attempts have been made to propose a sweeping unification of all the armed forces medical services into one integrated service. In fact the Civilian Health and Medical Program for the Uniformed Services (CHAMPUS) was a direct spin-off of these attempts.

Efforts to establish physical standards for selection and retention in aviation date back to World War One when Allied Forces were wrestling with integration of the airplane into normal operations. Recognizing the need for standardization in aviation medicine, Drs. Theodore Lyster and Isaac Jones developed a system of physical standards and education throughout the U.S. Army, which laid the foundation for the standardized approach to physical qualification for aviation service which we utilize today in all branches of the U.S. Armed Forces (6). Those early efforts of Doctors Lyster and Jones evolved into the mode off from in the millary, and as the Air Force broke for aviation medicine carred forvard But with five United States armed forces entering the modem era of avition, Aeromedical policies and physical standards have necessarily diverged to erational demands of the specific service.

Advances in technology drive the evolution of military tactics, which in turn results in changes to organizational structures, changes in Techniques, Tactics, and Procedures (TTPs) and the application of Combat Services Support on the battlefield. Changes in our current operational climate dictate the need to reconsider the practice of aviation med- icine in the military. Increasingly, Flight Surgeons and aircrew are deployed in settings where crewmembers must rely on Aeromedical support from sister service Flight Surgeons. Under current practices, Flight Surgeons are rarely trained and are even less frequently familiar with the aeromedica standards of their sister services. As a result, they will do their best to wade through the necessary bu reaucracy in order to meet the mission, frequently without achieving success and often failing to mee requirements. Differences in administrative frameworks between military aviation medicine program unnecessarily inhibit the Flight Surgeon's ability to provide needed services.

Proponents of unification of aeromedical systems across service boundaries tout an of administrion. These advantages may be particularly true during peacetime and in stationary military medical facilites. Opponents to unification rightly point out the differences in mission and operational environment between the services, and the requirement for medical assets organic to individual units that are able to provide optimal and timely care in these unique settings. Frequently, this requires divergence in training in order to provide an operationally relevant product.

While the authors do not necessarily advocate the unification of the medical services as a whole, the adoption of a common language and set of common tools within the aeromedical communities of the individual services has several advantages. Issues as simple as which form to use for a flight physical, or which labs to order, or as complex as how to classify an aeromedical dispos tion or how to process a waiver evaluation frustrate the Flight Surgeon and often lead to duplication of work, or worse. Standardization or increased concordance between the services would lead to more efficient and effective delivery of aviation medica che ual Fight Surgeon level

Clearly, each service has both shared (e.g. tion functions, and places unique physiological and psychologica stresses on the aviator. However, equally clear should be the reality that the vast majority of medical conditions (e.g. cardiovascular disease) will have the same implications in an aviator who straps into any aircraft, regardless of type or paint color. Evidence-based practice mandates that Flight Surgeons separate their aeromedical analysis from 
political service-based policy boundaries, and continue to delineate with the highest degree of fidelity which conditions, and to what degree these conditions will have a different impact on aviators operating from different platforms based on valid medical evidence. By and large, this work has lacked the broader perspective of military aviation medicine as a whole. Increasing collaboration and improved distribution of labor will lead to improved policies and improved Risk Mana tary aviation community.

Our vision is very simple. We are advocating and have been working in what has proven to be a very political realm towards the simple goal of having one system in which flight surgeons can experience true interoperabilty, providing aeromeditional reality and necessity which we believe can no canger be ignored.

end, in 2002, the authors began a series of posters, panel discussions and working groups presented at international conferences including the Aerospace Medical Association Scien-
tific Meeting and Medicine in Challenging Environments which brought together aviation medirine representatives from all of the U.S. Armed Services in order to discuss and further delineate the possibilities for improved collaboration in the development of Aeromedical policy and practices. As a result of these and many other sidebar discussions, we are happy to report several significant movements in the drection of Aeromedical jointness. In 2009 , the U.S. Navy, U.S. Army, and U.S. Coast Guard have adopted a common administrative framework

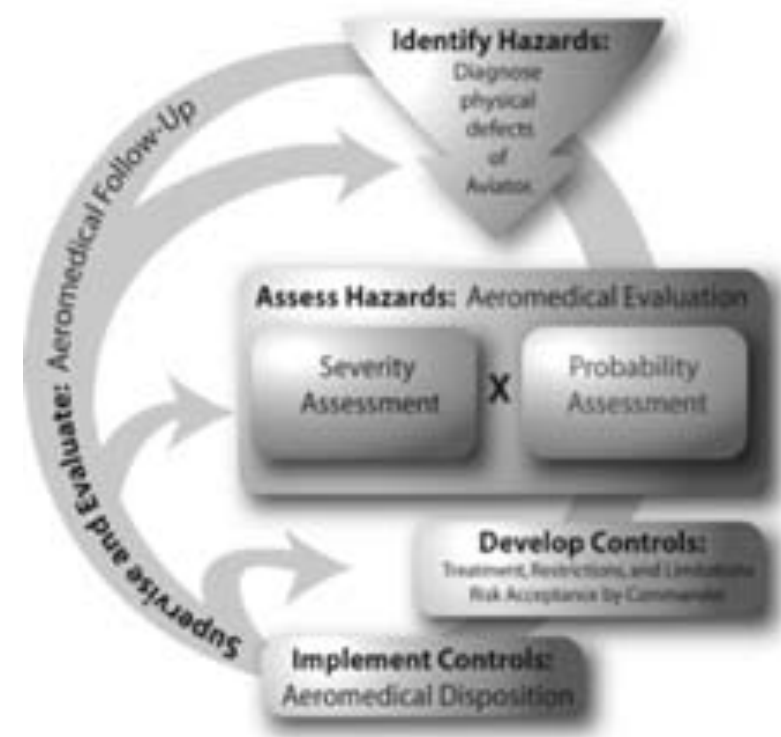

Figure 1: The Aeromedical Decision Making Process in the Aeromedical Epidemiological Research Office (AERO) for the workflow of flight physicals, and the U.S. Coast Guard and U.S. Army have agree o share a common system of physical standard ther special duty communities within the Army are also evaluating these systems as viable solution to parallel processes within those communites. While there is still significant work co do in the areas which wil be explored in this artocle, these landmark decisions mark a significant system of Aeromedical administration.

\section{AEROMEDICAL DECISION MAKING PROCESS} Critical to the process of joint aeromedica administration, must be a common system of ev ors Sauer and Woodson described the Aeromedical Decision Making Process (1) as an analog of cal Decision Risk medicine clinical and policy decisions.

The goal of the Aeromedical Decision MakProcess is to "prevent aviation mishaps due to physical or medical deficiencies... without unneces sarily restricting [military] aviation." It is the method that Flight Surgeons employ in order to evaluate specific conditions and crewmembers for entering or remaining on aviation service.

Within this framework, aeromedical policy and physical standards for aviation service are viewed as risk management controls to increase aviation safety. The effects of a given medica condilion must be evaluated on an individual and population basis in order to assess the impact upon severity and probability of contributing to a mishap or mission failure.

When applied to policy development, this process provides an objective means by which to evaluate the common Aeromedical concerns for a given medical condition which all sister services share, while attending to the specific differences in mission requirements free from the individual bias be noted that mission differences, rather than service differences, drive this aeromedical risk assessment precens process based on the real and observed aviation service-specific considerations for a specific mission or platform type far outweigh the differences.

The first hurdle to overcoming service boundaries in aviation medicine may very well be to adopt a common framework for the discussio and evaluation of aeromedical concerns. This model provides such a framework and may supply an efficient means for converting available medical evidence into better risk controls and aeromedical policies which serve all aircrew and flight surgeons regardless of nationality or service membership.

\section{DARDS}

Each of the U.S. armed services enjoys its own unique culture and challenges. These may range flom simple diferences in language to more complex significant mission requirements such as ang ing an aircraft carrier landing. An effective joint system for aeromedical administration must account for these differences. Before moving forward with any type of program implementan, we must firt present. As this question has been analyzed, the authors have identified four primary hurdles: 1) difference in terminology including aviator classification 2) a difference in miscion definitions and requirements, 3) a difference in the processes of policy development, and 4) a difference in the review and application of those policies.

\section{DIFFERENCES IN TERMINOLOGY}

Individual service cultures and administrative landscapes have contributed to the development of non-standard terminology in aviation medicine. While the meaning in most cases translates in the same manner, it is difficult for members of one service to understand a sister service's policy stance for no other reason than differences in language. Before moving forward with common policy minology or "common language.

\begin{tabular}{|c|c|}
\hline Air Force & Navy \\
\hline $\begin{array}{l}\text { Flying Class I: Selection for Pilot } \\
\text { Training } \\
\text { Flying Class IA: Selection for } \\
\text { Navigator Training } \\
\text { Flying Class III: non-rated duties } \\
\text { Categorical Flying Class II } \\
\text { - FC IIA: Low-G aircraft } \\
\text { (tanker, transport, } \\
\text { bomber) } \\
\text { - FCIIB: Non-ejection Seat } \\
\text { - FCIIC: Specified } \\
\text { restrictions }\end{array}$ & $\begin{array}{ll}\text { Class 1: } & \text { Pilots (Naval Aviators) } \\
\text { - } & \text { Service Group I: } \\
\text { unrestricted (including } \\
\text { night carrier operations) } \\
\text { - Service Group II: no } \\
\text { shipboard operations } \\
\text { (except helicopter) } \\
\text { - Service Group III: } \\
\text { dual-control only; with } \\
\text { SG I/II copilot } \\
\text { Class 2: All other aircrew (Naval } \\
\text { Flight Officer, Flight Surgeon, etc.) } \\
\text { Class 3: Air Traffic Controllers, UAV } \\
\text { operators, etc. }\end{array}$ \\
\hline
\end{tabular}

Army/Coast Guard Class 1A: Initial pilot applicant Commissioned) Class 1W: Initial pilot applicant (Warrant) Class 2: Rated aviator Class 2F: Flight Surgeon, Class 3: All other aircrew (crew chiefs, gunners, flight medics, aer observers, maintenance aircrew, altitude chamber technicians, UAV operators)
Class 4: Air Traffic Controllers
The wide variation in terminology used illustrates our incongruities. Even simple concepts

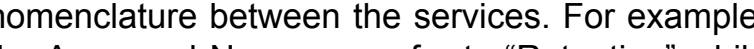
aviation as "Not Qualified" while the Army refors the same aviator as "Disqualified" and the Navy as "Norce will describe physical limitations army and Air ed Duty." Similar terminology differences abound

in normally inferred. The important thing to recognize cultural aviation medicine environment. More importantly, such language will have to migrate toems are developed.

More troubling than differences in language Army/Coast Guard, Navy, and aches are immediately evident (Table 1). Aeromedical policies avident (Table 1). 
Aeromedical disposition in each service is grounded in its own aeromedical classification system, each of which have developed through an amalgam of service culture and regulatory framework entirely outside the realm of aviation medicine. It is fairly easy to recognize that these classes are defined in each service based on fundamentally different frameworks. In many cases, the difficulty in interpreting aeromedical physical standards is rooted the differences in these classification systems.

It is difficult to see a truly joint aeromedical system that uses the current service-specific aircrew classification structure. Standardization of this system into a common inter-service aircrew classification would seem the only plausible solu-

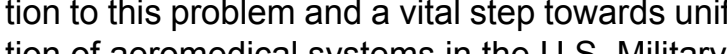

Several solutions may present themselves, but one potential solution could be based upon crewmember type and basic tion. There are only four essential types of individuals who require aeromedical clearance, each of which represents unique job-related physical quirements: 1) flight crew who control aircraft , 2) flight crew who do not control aircraft 3) crewmembers who perform ancillary duties in flight (aerial observers, weapons system operators, equipment operators, etc) unrelated to the control of the aircraft 4) individuals who perform flight-related duties, but not involving actual flight duties (ground crew, ATC, UAS operators etc). Accordingly, one potential interservice classification system might look like ingt seen in table 2a, which separates mission specific considerations and initial vs. retention considerations from the basic element of disposition clas(a) risk to aeromedical threat (Table $2 \mathrm{~b}$ ).

\begin{tabular}{|c|c|}
\hline Aircrew Class & Description \\
\hline Class A & Non-flight crew performing aerial duties \\
\hline Class B & Non-flying, flight related personnel \\
\hline Class $\mathrm{C}$ & Flight Crew, Pilot in Control, single-control aircraf \\
\hline Class D & Flight Crew, Pilot in Control, dual-control aircraft \\
\hline Class E & Flight Crew, Non-pilot \\
\hline
\end{tabular}

\begin{tabular}{|l|l|l|}
\hline & In-Flight Crew & Ground-based \\
\hline Flight Critical & Class A & Class C \\
\hline Flight Important & Class B & Class D \\
\hline \multicolumn{2}{|l}{} \\
\hline
\end{tabular}

2b: Proposed Interservice Aircrew Classification System

version 2 version 1
Any classification system will require the Flight Surgeon to make decisions based on individual crewmembers and their specific job requirements, but an effective classification system must account for differences in physiological requirements. The key point is that adoption of a comervice classification structure such as that presented in tables $2 \mathrm{a}$ or $2 \mathrm{~b}$ would facilitate cross-service communication and allow for a common framework of aeromedical regulations, moving unified joint aeromedical system.

\section{RAMEWORK FOR STANDARDS DEVELOP}

\section{(MSSION VS. PHYSIOLOGY)} missions may be categorized based upon , hese distinct missions (e g land-based vs. carrier-baced in the Navy), the platform flown (e.g. fixed vs, roary wing) or the complement of crew (e.g singpilot vs. multi-crew aircraft). However, each type of aviation platform places its own unique set of physical demands on the aviator, while many demands are common to all aviation platforms.

Aeromedical concerns are more appropriately described in reference to the mission the aircrew member is serving than to the branch of service of which he or she is a member. There is no doub that due diligence must be paid to the physiological demands of specific missions and equipment on the crewmember. It must be noted, however, that these demands are grouped into categories that transcend service boundaries. The present system effectively prevents aeromedical categorization of missions across the services. More importantly, it frequently does not even account for actual physical stresses on different categories of crew members within a given service. An effective framework for aeromedical standards would approprately acent for discrion, equipment, envionment and other job requirements.

One example of the failure to conside mission specific physical demands is ilustrated by looking at the standards for stereopsis in Army airsis for crewmembers (non-pilots) but not for pilots $(9,11)$. This may represent a leftover policy from the primarily fixed wing days of Army aviation medicine and is probably based on reasonable rationale: pilots need advanced stereopsis on final approach and landing phases of flight which are within the stereoptic range, and back-end helicopter crewmembers' duties would not routinely call on their stereoptic capability as they manage payload. Yet evidence supports the idea that monocular pilots (without stereopsis) do just as well as binocular pilors in landing aircraft $(15,16)$ and mission analysis really demonstrates that the army crewmember may require greater degrees of stereopsis than the front end crew as he controls aircraft position in fine detail during air assault, fast-rope, sling-load, and routine hot-loading operations. The basic (reasonable) premise for this inversion of standards is most likely based on a general impression that pilots rethan non-pilots.

This is a seemingly simple oversight, but it highlights the difficulties encountered when we fail to consider the actual physical demands of mission phenomenon by comparing the differences between high-Gz platforms, carrier-based landings, rotary wing platforms, and Unmanned Aerial Systems (UAS) Other critical differences in demands differences in operating altitude and G-Forces. One approach to developing a function jint system would be to examine the unique physiological aspects of broad categories of aircraft (Table 3).

\begin{tabular}{|c|c|c|c|}
\hline Rotary Wing & $\begin{array}{l}\text { Fixed Wing - Low Gz } \\
\text { (tanker, transport, bomber) }\end{array}$ & $\begin{array}{l}\text { Tactical Jet - Hi } \\
\text { Gz }\end{array}$ & $\begin{array}{l}\text { Unmanned Aerial } \\
\text { Systems (UAS) }\end{array}$ \\
\hline 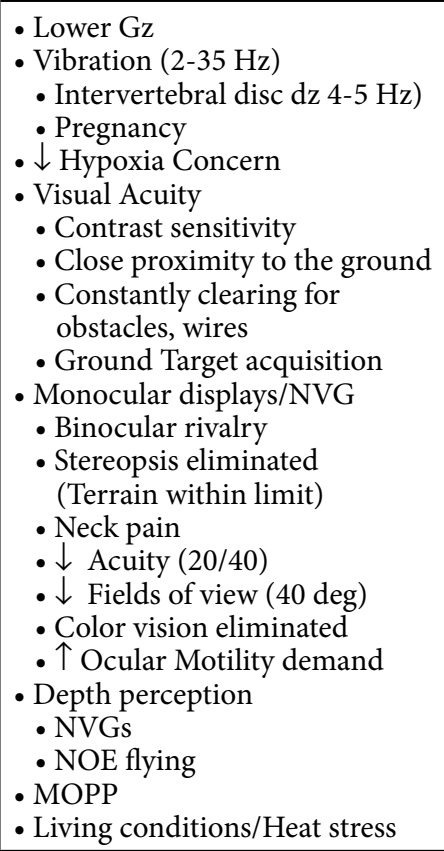 & $\begin{array}{l}\text { - Lower Gz } \\
\text { - Long Duration Missions } \\
\text { - Large Crews, often dual } \\
\text { piloted } \\
\text { - } \uparrow \text { Hypoxia Concern } \\
\text { - Stereopsis less critical } \\
\text { - Decompression (also } \\
\text { pressure suits) } \\
\text { - Radiation (high altitude } \\
\text { recon) } \\
\text { - } \uparrow \text { Circadian rhythm shifting }\end{array}$ & $\begin{array}{l}\text { - High, rapid-onset Gz } \\
\text { - } \uparrow \uparrow \text { Hypoxia Concern } \\
\text { - Long Duration Missions } \\
\text { - Decompression } \\
\text { - Visual Acuity } \\
\text { - Increased visual demand } \\
\text { for tactical mission } \\
\text { - Close proximity to the } \\
\text { ground } \\
\text { - Air Tanget acquisition } \\
\text { - Ejection Seats }\end{array}$ & \begin{tabular}{|l} 
- Ground Based \\
- No Hypoxia Concern \\
- No pressure differentials \\
- Color Vision critical \\
- thereopsis not required \\
- Dual Pilot \\
- Potential for in-flight crew \\
changes \\
- Increased demands on \\
decision making and \\
situational awareness \\
challenget traditional views \\
of UAS requirements \\
- Vlying in Class A \\
Airspace \\
- Weapons and targeting \\
systems
\end{tabular} \\
\hline
\end{tabular}

When examining physical standards from physical standards emerges; one which would serve the needs of all services, and would be based on physiological demands rather than politica boundaries. A very important consideration is that each service does not necessarlly operate within ts own traditional boundaries; missions traditionally reserved for one service may be conducted by differe from another service. One commonly cited diference is the Navy requirement for carrier-based Yet Army, an obviously demanding aviation task. Yving, Coast Guard, and Air Force rotary wing platforms. Current tructure would not account for these challenges address platform and mission based differences, new joint aromedical structure would allow us a dence based approach to aviation medicine.

An aeromedical evaluation of an aviator could be made in the context of platform/mission based parameters. A pilot, flight officer or aircrew member can be effectively authorized or restricted to fly on different types of missions, based on the this perspective, a more suitable framework for

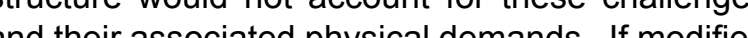
service boundaries and would better reflect an evi- 


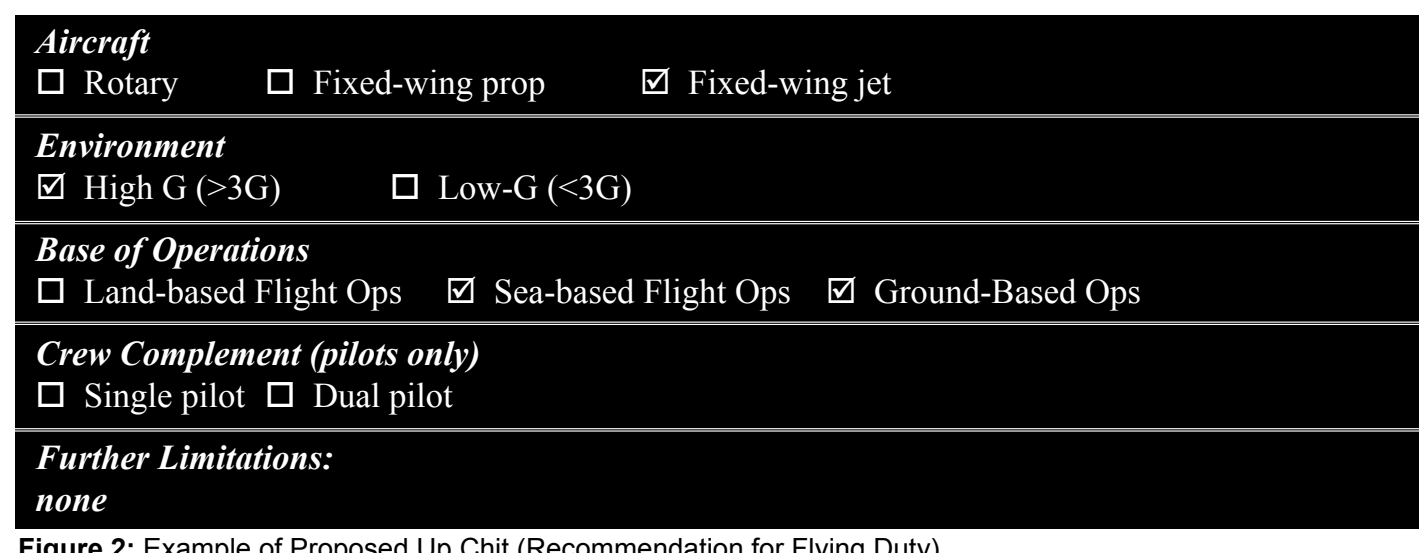

corresponding aeromedical stressors and that person's physical and mental capabilities. More apthat the service member is fully qualfied fored so egories except for those indicated. One example of this classification, as it might appear on a joint aeromedical clearance chit (up slip) is seen in Figure 2 . Such a system would allow classification of pilots, flight officers and enlisted crewmembers, re gardless of service, based upon physical and mental capabilities from an aeromedical perspective. With the increasing incidence of exchange tours, particularly among pilots between the services, this system would allow a common basis of categorization, further helping to eliminate the "language barriers" that exist between the aeromedical branches of the Army, Navy and Air Force.

DIFFERENCES IN THE PROCESSES OF POLICY DEVELOPMENT

Currently, each service maintains parallel analogous organizations which develop and implement aeromedical policy (Code 42, Army Aeromedical Activity (AAMA, Aeromedical Corporate Board, Aeromedical Consult Service, Aeromedical Advisory Council, etc). Each service also maintains its respective process for submission, review, and disposilon of aecoms the subtandards as well as policy culy aviation medicine.

As we consider the convergence of aeromedical systems, each service must ensure that its administrative eacromedical system ensure that serve its own interests. Migration towards a common process and waiver guide is a step-wise approach and must ensure that these interests and representation are maintained. The authors do not advocate for or suggest a radical course change, but rather a common effort towards commonality. We have already begun to work toward this end an oday's aeromedical environment, waiver policies are usully modified with at least some modicum of collaboration between the services, Joint policies are in some cases being adopted, and convergence into common electronic systems is evolving. (Some of these will be discussed later in this article.) deally, however, services could eventually move (when collectively ready) towards some system of formal "joint aeromedical council" or board which could manage a truly joint aeromedical waiver guide and/or disposition system. This idea may seem alarming to some, but the important thin to recognize is that there is an entire spectrum of possiblities to consider, including formal and infor-a mal processes and systems. In order to sustain a joint process we will need to establish some kind of mechanism by which Aeromedical policies are developed, considered, implemented, and modified while protecting the interests and concerns of all the services. While clearly not comprehensive pproach which could move us carefully in the joint direction.

\section{DIFFERENCES IN REVIEW AND APPLICATION} PROCESS

While medical conditions may be interpreted differently by each service, or in aircrew members flying different mission platforms, most Aeromedical Physical Exam requirements are (and should be) based on sound medical/public health screening principles, and should not vary by service or missio

One of the easiest and highest yield obsta作 requirements for initial and periodic aeromedica evaluations across services and mission platforms. Wading through the service-specific regulations and instructions, we identify a grossly incongruent set of physical exam requirements. Differences exist in issues as simple as who is required to undergo ECG testing, or who must have a G6PD, urinalysis, or lipid panel. Chest $X$-rays do not share common mandates and each service has its own variation of anthropometric testing and cardiac risk profiling. Yet, we all seek the same outcome. Most of these differences probably reside not in the medical merit of the tests themselves, but in the differences in policy development as outlined previously. Yet these difterences are extensive, they are the culprit in wasling numerous man-hours when an aviator ical with a sisterservice flight surgeon wich does not meet his service standards.

Adoption of a singl (t) tablished aircrew members should be fairly simple to achieve and would represent tremendous progto achieve and would represent tremendous prog-
ress toward commonality. The net effect of this one change would be a dramatic improvement in interoperability of the flight surgeon in the joint environment. Another hurdle which presents a fairly simple opportunity for convergence is found in the paperwork drill. In spite of Department of Defense level efforts to standardize the physical exam forms

\section{nitial Steps}

Individual services move toward common "best-practices" as aeromedical policies (waiver guides) come up for review:

informal collaboration across services (information sharing) with goal as unified approach to a given aeromedical condition. Basic physical exam requirements (exam, labs, forms, etc) are unified (see above).

Aviator Classification system is unified (see above).

Cross-pollination in training (joint residencies) and joint assignments at aeromedical centers, leading to better information sharing and opportunities for collaboration.

\section{(ical Council"}

Joint forum in which representatives from all the services and aviation communities are able to share ideas and information in a unified effort to develop congruent "best practices" in waiver policies across service boundaries. Strategic long-term plan to review all aeromedical waiver policies in systematic manner over time.

Barriers to commonality in waiver policy, administrative requirements, etc are explored and ultimately problems are solved.

\section{Consolidated Joint Waiver Guide}

Policies are unified through an evidence-based risk management model, which accounts for all mission/service

needs under a unified classification mode.

roved product without unnecessary duplication of effor

All Flight Surgeons have a single tool that allows for improved management of aviators in an increasingly joint environment. Table 4: Policy Development and Implementation of Process the DD2807 and DD2808, we have not seen uniup chit described previously, adoption of (to utilize 作 vice cultures. of aeromedical evaluations. Each service reTraditionally, physical exams were (1) corresponding adminisworld-wide-web technology has presentdhe possibility for a new model for review and gether. A common internet application shared by ervice authorities would better facilitate crossand provide for a common process which would and research. better facilitate joint aeromedical communication 
THE AEROMEDICAL ELECTRONIC RESOURCE OFFICE (AERO) AND CURRENT JOINT INITIATIVES AS AN EXAMPLE OF CONVERGENCE

Opportunities to converge towards common systems, policies, and practices abound and do not necessarily require a monumental overhaul of what is currently in place. While compromise is important, the service-specific aeromedical authorities do not need compromise on their standards or requirements in order to find common ground. One example of such endeavors can be found in recent developments in the integration of the Aeromedical . Alabama.

In 2002, the U.S. Army Aeromedical Activity (USAAMA) adopted AERO as an internet-based placing a cumbersome paper-based submission process in the Army. This governmed submission developed system was fielded, and over a short period of time, resulted in significant improvements in the disposition of Army flight physicals. In addition to improving the submission process, internalition cessing times at USAAMA were reduced from 150 days to 1-2 days on average, while also making making AERO provided for data checking and was easily integrated into the Flight Surgeon's office, both CONUS and OCONUS in the deployed setting. Administrative errors on submitted physicals were reduced from $40 \%$ on the paper-based system to $<1 \%$ on AERO, and immediate feedback was provided to the Flight Surgeon on the disposition of aircrew physicals. Backlogs were cleared and overall efficiency was dramatically improved.

In 2008, with problems similar to those experienced using the Army's paper-based systems, steps were taken independently in the U.S. Navy and U.S. Coast Guard to implement AERO as their system for aeromedical disposition and review. While still undergoing testing and implementation in the potential to make significant improvemen has the protent io disposition and allows for coments in ity on an entirely different level than frer before.

There are several points about this AERO igration which must be emphasized. Firstly, the Army, Coast Guard, and Navy all shared a similar pathway for review and disposition within their own organizational structures (e.g. all three use a centralized review authority). Secondly, the Coast Guard and Army share a common footing in aeromedical culture as a result of the sharing of a common training base for Flight Surgeons, and a common migration of Army Flight Surgeons into the Coast Guard medical service. Thirdly, in the case of the Coast Guard, the aeromedical physical exam parameters (items required for physica exams) were already very similar. To cement these similarities, the Coast Guard agreed to adopt the same standards utilized by the Army, and Code 42 in the Navy has worked diligently to more closely align physical exam parameters with the Army and Coast guard in order to facilitate AERO integration. The opportunity presented by Navy and Coast Guard AERO integration allowed AAMA to make some minor modifications to AERO to acvy's aeromedical policy. Without modification, vy's aeromedical policy. Without modification, the process of review within AERO very easily accomservices. Because AERO utilizes a role based system, the Becauseaiver authority could be redained in the service and allow for service-specific review while allowing all three services to utilize a common system and begin to migrate specific physical exam parameters, beginning to overcome one of the previously mentioned hurdles to commonality.

While AERO is only one system and on example, it serves the purpose of this article, as a vivid example of the capacity for convergence towards the authors' vision of a single common aeromedical system, while simultaneously raising the program standards within each individual service.

\section{CONCLUSION}

The prospect of developing a joint Aeromedical System and Waiver Guide is clearly daunting and is not without its challenges. Service culture, existing systems, policies, and service specific regulatory landscape all play important roles in keeping aeromedical systems separate. It is clear with the continuing evolution of the military operational environment that each of the services mus work to migrate towards commonality while time pernis, before higher authes jove. In the meantime, the benefits of increased interperability, and the facilitation of a romed epidemiological research. Despite aeromedica divergence over the course of the last 100 years since the beginning of military aviation medicine we are beginning to see a convergence towards a common system. As we continue to explore and overcome hurdles to joint aeromedical systems, we will see that the authors' vision of a single common system of Aeromedical Administration for the U.S. Military is indeed within reach.
REFERENCES

Sauer S, Woodson J. The Aeromedical Decision Making Process. Society of US Army Flight Surgeons Newsletter 2002;5(1):1, 13-16.

Woodson J, Thomas D. Rotary Wing Aeromedical Standards Compared. 2002 May 7; Montreal.

3. Dalitsch W, Woodson J. Overcoming Obstacles in the Development of Tri-Service Aviation Physical Standards. 2003 May 6; San Antonio.

4. Woodson J, Dalitsch W, Parsa B, Harman K, Bernstein $S$, Ciccone C. Quad-Service Standards: It's Time to Make it Happen. Aviation, Space, and Environmental Medicine 2009;80(3):243-4.

5. Woodson J, Dalitsch W. Overcoming Obstacles in the Development of Tri-Service Aviation Physical Standards. 2009 Feb 3-5; Galveston, TX.

6. Jones IH. Flying Vistas: the Human Being as Seen Through he Eyes of the Flight Surgeon. J. B. Lippincott Company;

Van Syoc D. Aerospace Medicine Practice Guidelines. Aerospace Medical Association 73rd Scientific Meeting. Montreal; 2002 .

Justin Trevor Woodson (MD, MPH) is a Lieutenant Colonel in the United States Army. He is currently a faculty member in the Department of Military and Emergency Medicine and fellow in Military Medical History at the Uniformed Services University of the Health Sciences (USUHS) in Bethesda, MD. LTC Woodson is a Flight Surgeon and a Dive Medical Officer (DMO) and is Board Certified in Aerospace Medicine. LTC Woodson's primary career emphasis has been in applied operational medicine in unusual environments and he enjoys a diverse training background experience and hands-on discovery.

Walter Dalitsch III (MD MPH) is a Navy and Marine Corps aerospace medicine specialist who has just completed a three-year tour as the faculty flight surgeon for the Navy's School of Aviation Safety in Pensacola, Florida. He has assumed duties as the Force Surgeon for the Naval Air Forces, Pacific Command, headquartered in San Diego. Prior to the Navy he worked as the daytime emergency room physician and nighttime radio deejay in the Alaskan Yup'ik Eskimo village of Bethel. He has published and presented extensively on the history of aviation medicine, is a life member and Fellow of the Aerospace Medical Association, and enjoys flying in his spare time.

James S. McGhee (MD, MPH) is certified in aerospace medicine and is the senior consulting flight surgeon for the US Army Aeromedical Activity, Fort Rucker, AL. He earned his medical degree from the Medical College of Virginia and his public health degree from the Johns Hopkins University. He completed a distinguished career in the United States Army serving in a variety of capacities including Dean of the US Army School of Aviation Medicine, Commander of the US Army Aeromedical Research Lab and Consultant to the Army Surgeon General for Aerospace Medicine. Prior to his medical career, he earned a master's degree in environmental engineering and worked for the US Navy. He has authored a number of publications, principally in the field of aerospace medicine. 